\title{
PROPOSED REVISION OF THE STAGING CLASSIFICATION FOR ESOPHAGEAL CANCER
}

Robert J. Korst, MD

Valerie W. Rusch, MD ${ }^{\mathrm{a}}$

Ennapadam Venkatraman, $\mathrm{PhD}^{\mathrm{b}}$

Manjit S. Bains, MD

Michael E. Burt, MD, PhD

Robert J. Downey, MD ${ }^{\mathrm{a}}$

Robert J. Ginsberg, MD ${ }^{\mathrm{a}}$
Objectives: This study analyzed survival with respect to lymph node involvement to develop a new staging system for patients with esophageal cancer that accurately reflects prognosis. Methods: The records of patients undergoing resection of primary esophageal cancer from 1989 to 1993 were reviewed. The data collected included patient age and sex, tumor histologic characteristics and location, the use of preoperative or postoperative radiation and chemotherapy, the type of resection, the depth of tumor invasion, the number and location of benign and malignant lymph nodes in the resected specimen, the disease status at last follow-up, and the first site of relapse. With an anatomically specific lymph node map, tumors designated in the current American Joint Committee on Cancer system as M1 because of extensive lymph node metastases were reclassified as N2, reserving the M1 category for visceral metastases. Survival was analyzed by the Kaplan-Meier method, and prognostic factors were assessed by logrank and Cox regression analyses. Results: There were 216 patients (159 men, 57 women) with a median age of 63.5 years. Adenocarcinoma of the distal esophagus or gastroesophageal junction was the most common tumor (127 patients, 59\%) and Ivor Lewis esophagogastrectomy was the most frequently performed operation. Both lymph node location (N1 versus N2) and number ( 0 vs 1 to 3 vs 4 or more) significantly influenced survival. Conclusions: A new staging system that adds an N2 M0 descriptor and reclassifies stage groupings reflects prognosis more accurately than does the current American Joint Committee on Cancer staging system. The number of positive lymph nodes is also an important stratification factor. (J Thorac Cardiovasc Surg 1998;115:660-70)
$T^{\text {then }}$ he current American Joint Committee on Cancer $(\mathrm{AJCC})^{1}$ staging system for carcinoma of the esophagus is TNM based. $T$ (1 through 4) represents progressive invasion of the tumor into the esophageal wall, $N$ reflects the presence (N1) or absence (N0) of regional lymph node metastasis, and $M$ designates the presence (M1) or absence (M0) of

From the Thoracic Service, Department of Surgery, ${ }^{\text {a }}$ and the Biostatistics Service, Department of Epidemiology and Biostatistics, ${ }^{\mathrm{b}}$ Memorial Sloan-Kettering Cancer Center, New York, N.Y.

Read at the Twenty-third Annual Meeting of The Western Thoracic Surgical Association, Napa, Calif., June 25-28, 1997.

Received for publication July 1, 1997; revisions requested Oct. 13, 1997; revisions received Nov. 14, 1997; accepted for publication Nov. 17, 1997.

Address for reprints: Valerie W. Rusch, MD, Memorial SloanKettering Cancer Center, 1275 York Ave., New York, NY 10021.

Copyright (C) 1998 by Mosby, Inc.

$0022-5223 / 98 \$ 5.00+0 \quad \mathbf{1 2 / 6 / 8 7 6 8 2}$ distant metastasis. The M1 category includes both visceral organ metastasis and lymph node metastasis beyond the nodes that are considered "regional."

The adequacy of the current staging system has been questioned for several reasons. First, it does not fully consider tumors located at the gastroesophageal junction. Second, it includes only general anatomic descriptions of which lymph nodes are "regional" and which represent more extensive nodal disease. Third, by considering lymph node involvement outside of the regional nodes to be M1, it classifies such tumors as stage IV and categorically unresectable. Finally, it does not stratify according to the number of metastatic lymph nodes, a factor thought to be important for predicting survival of patients undergoing resection for esophageal carcinoma.

The perceived inadequacies of the current staging system prompted us to review our experience with patients undergoing surgical resection of primary esophageal cancer. The objectives of this study were 
to classify lymph node involvement according to an anatomically specific map, to analyze survival with respect to the location and number of involved lymph nodes, and to develop a staging classification that accurately reflects prognosis in patients with esophageal cancer.

\section{Methods}

The records of all patients undergoing surgical resection for primary esophageal carcinoma on the Thoracic Service at Memorial Sloan-Kettering Cancer Center from 1989 to 1993 were reviewed. This allowed a minimum follow-up of 3 years. Patients were excluded from analysis if adequate follow-up information could not be obtained by physician visit or telephone contact with the patient, family, or primary physician. The data collected included patient age and sex, tumor histologic characteristics and location, the use of preoperative or postoperative radiation and chemotherapy, the type of resection, the depth of tumor invasion ( $T$ descriptor), the number and location of benign and malignant lymph nodes in the resected specimen, the disease status at last follow-up, and when appropriate the first site of relapse.

The $\mathrm{T}$ descriptors used were those defined by the current AJCC staging system. ${ }^{1} \mathrm{~N}$ and $\mathrm{M}$ status were also first designated according to this system, with positive regional lymph nodes classified as $\mathrm{N} 1 \mathrm{M} 0$ and positive lymph nodes extending beyond the regional area classified as N1 M1. The process of classifying regional lymph nodes was done by systematic review of operative summaries and pathology reports, applying a proposed lymph node mapping system (Fig. 1) that provides a number for each lymph node group by anatomic location. ${ }^{2}$ This map has also been used in recent clinical trials examining the treatment of resectable esophageal carcinoma. As in the AJCC staging system, any patient with visceral metastasis was also classified as having M1 disease. For the purposes of a "revised" staging system, patients who had M1 disease because of positive lymph nodes were reclassified as having N2 M0 rather than N1 M1 disease (Table I). The revised staging system allowed separate analysis of survival in this cohort of patients with more extensive nodal disease but without visceral metastasis.

Overall survival was calculated from the date of operation and described with the product limit method of Kaplan and Meier. ${ }^{3}$ Overall and disease-free survivals were compared across the levels of prognostic factors with the log-rank test. ${ }^{4}$ Multivariate analyses were performed by Cox regression. All statistical tests were two-sided.

\section{Results}

During the 5-year period from January 1989 through December 1993, 233 patients underwent esophagectomy. Follow-up information until death or for a minimum of 3 years was available for 216 patients. These patients, 159 men and 57 women with a median age of 63.5 years (range 31 to 85 years), form the basis of this analysis. The histologic characteristics and anatomic locations of the primary tumors are shown in Table II. Most patients $(59 \%)$ had adenocarcinomas in the distal third of the esophagus or at the gastroesophageal junction. The types of operations performed and the frequencies of preoperative and postoperative adjuvant therapies are also shown in Table II. Ivor Lewis esophagogastrectomy was the most frequently used procedure ( $70 \%$ of patients). Thirty-two patients $(15 \%)$ received preoperative treatment, and 28 patients $(13 \%)$ received adjuvant therapy. A total of 167 patients $(77 \%)$ did not receive any treatment other than esophagectomy. This series thus largely reflects the outcome of patients treated by surgical resection alone.

At last follow-up, 76 patients remained free of disease. Information about the initial site of relapse was not available for 21 patients who either had died of disease or were alive with recurrent cancer. The sites of first relapse in the remaining 119 cases are shown in Table III. Distant metastases were the most common initial sites of recurrent disease, occurring in approximately $40 \%$ of patients. Local and regional recurrences were equally frequent, seen in $16 \%$ to $21 \%$ of patients with recurrence. Tumor histologic characteristics did not influence the first site of recurrence.

The overall survival curves by TNM status are shown in Fig. $2(A)$. The revised TNM classification is shown in parentheses. Tumors designated as N1 M1 in the current AJCC system because of lymph node metastasis beyond the regional nodes would be classified as N2 M0 in the revised staging system. There were no significant differences in overall survival between $\mathrm{T} 1 \mathrm{~N} 0 \mathrm{M} 0$ and $\mathrm{T} 2 \mathrm{~N} 0 \mathrm{M} 0$ tumors $(p=0.27)$ or between T3 N0 M0 and T1 through T3 N1 M0 tumors $(p=0.68)$. However, patients with T1 through T2 N0 M0 tumors had a significantly better survival than did patients with T3 N0 M0 and T1 through T3 N1 M0 tumors $(p<0.01)$. In turn, these patients had a better survival than did patients with N2 M0 tumors $(p<0.001)$. The number of T1 through T2 N2 M0 tumors was too small $(n=10)$ to permit a comparison with T3 N2 M0 tumors. When considered alone, the survival of patients with T3 N1 M0 tumors was significantly better than that of patients with any N2 M0 tumor $(p=0.006)$. The six patients who had T4 tumors appeared to have the worst survival, although these results must be interpreted with caution because of the small number of patients in this category. It thus appears that TN 

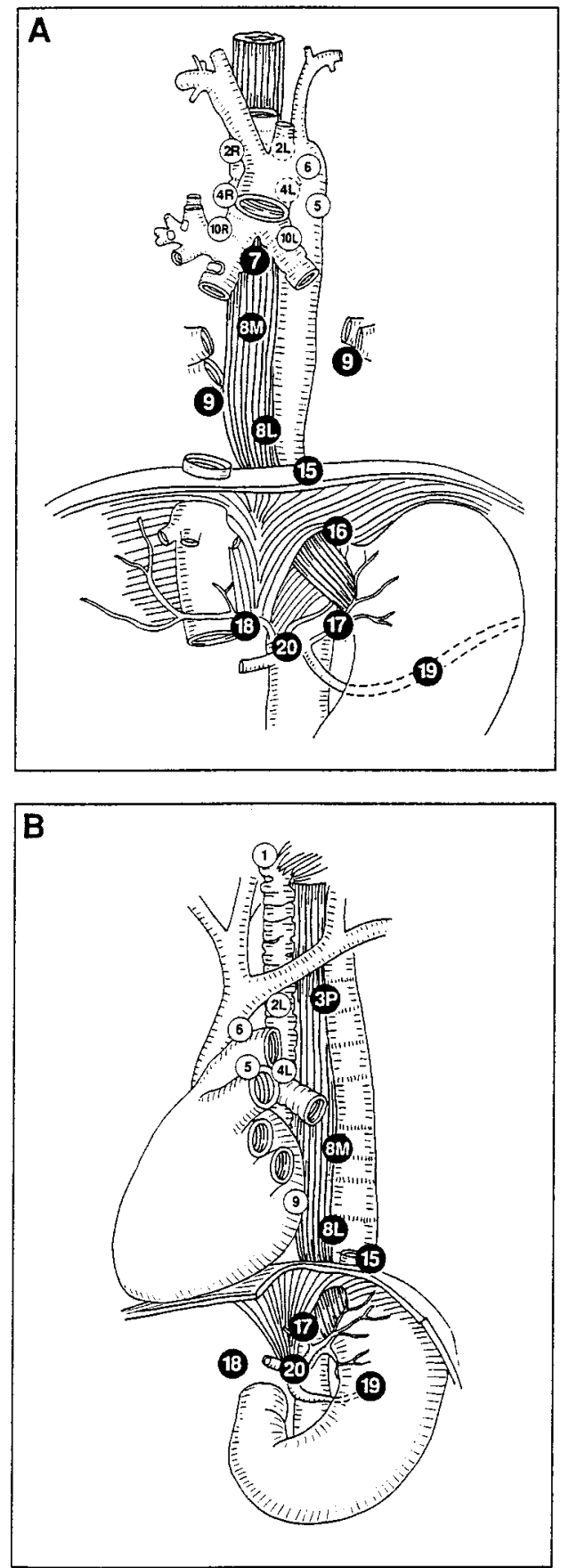

Regional lymph node stations for staging esophageal cancer, from front $(A)$ and side (B).

\section{STATION/DESCRIPTION}

1 Supraclavicular nodes

2R Right upper paratracheal nodes

2L Left upper paratracheal nodes

3P Posterior mediastinal nodes

4R Right lower paratracheal nodes

4L Left lower paratracheal nodes

5 Aorto-pulmonary nodes

6 Anterior mediastinal nodes

7 Subcarinal nodes

8M Middle paraesophageal lymph nodes

8L Lower paraesophageal lymph nodes

9 Pulmonary ligament nodes

10R Right tracheobronchial nodes

10L Left tracheobronchial nodes

15 Diaphragmatic nodes

16 Paracardial nodes

17 Left gastric nodes

18 Common hepatic nodes

19 Splenic nodes

20 Celiac nodes

\section{LOCATION}

Above suprasternal notch and clavicles

Between intersection of caudal margin of innominate artery with trachea and the apex of the lung

Between top of aortic arch and apex of the lung

Upper paraesophageal nodes, above tracheal bifurcation

Between intersection of caudal margin of innominate artery with trachea and cephalic border of azygos vein

Between top of aortic arch and carina

Subaortic and para-aortic nodes lateral to the ligarnentum arteriosum

Anterior to ascending aorta or innominate artery

Caudal to the carina of the trachea

From the tracheal bifurcation to the caudal margin of the inferior pulmonary vein

From the caudal margin of the inferior pulmonary vein to the esophagogastric junction

Within the inferior pulmonary ligament

From cephalic border of azygos vein to origin of RUL bronchus

Between carina and LUL bronchus

Lying on the dome of the diaphragm, and adjacent to or behind its crura

Immediately adjacent to the gastroesophageal junction

Along the course of the left gastric artery

Along the course of the common hepatic artery

Along the course of the splenic artery

At the base of the celiac artery
Stations 11 (interlobar), 12 (lobar), 13 (segmental), and 14 (subsegmental) are intrapulmonary nodes and have been exciluded.

RUL: right upper lobe

Fig. 1. Lymph node mapping system for esophageal cancer. $R U L$, Right upper lobe; $L U L$, left upper lobe. Stations 11 (interlobar), 12 (lobar), 13 (segmental), and 14 (subsegmental) are intrapulmonary nodes and have been excluded. (From Casson AG, Rusch VW, Inculet RI, Ginsberg RJ, Zankowicz N, Rosenberg JC, et al. Lymph node mapping for resectable carcinoma of the esophagus: a guide for thoracic surgeons. Princeton (NJ): Bristol-Myers Oncology Division; 1992. Reproduced with permission.) 
Table I. Definitions of N1 versus N2 lymph node stations used in defining TNM status for the revised staging system for esophageal carcinoma

\begin{tabular}{lll}
\hline \multicolumn{1}{c}{ Tumor location } & \multicolumn{1}{c}{ N1 lymph nodes } & N2 lymph nodes \\
\hline Cervical & Levels 1,2 & All other involved nodes beyond N1 \\
Thoracic, upper third & Levels $1,2,3,4,7,10$ & \\
Thoracic, middle third & Levels $2,3,4,7,8,9,10$ & \\
Thoracic, lower third & Levels $7,8,9,11,15,16$ & \\
Gastroesophageal junction & Levels $9,15,16,17,18,19,20$ & \\
\hline
\end{tabular}

Table II. Clinical characteristics of 216 patients with esophageal carcinoma

\begin{tabular}{|c|c|c|c|}
\hline & & No. & $\%$ \\
\hline \multirow{3}{*}{ Tumor histology } & Adenocarcinoma & 127 & 59 \\
\hline & Squamous cell carcinoma & 84 & 39 \\
\hline & Other & 5 & 2 \\
\hline \multirow[t]{6}{*}{ Tumor location } & Cervical & 2 & 0.9 \\
\hline & Thoracic, upper third & 3 & 1.4 \\
\hline & Thoracic, middle third & 52 & 24 \\
\hline & Thoracic, lower third & 59 & 27 \\
\hline & Gastroesophageal junction & 98 & 45 \\
\hline & Multifocal & 2 & 0.9 \\
\hline \multirow[t]{6}{*}{ Type of operation } & Bypass, no resection & 1 & 0.4 \\
\hline & Transhiatal & 45 & 21 \\
\hline & Ivor Lewis & 150 & 70 \\
\hline & Left thoracotomy & 7 & 3 \\
\hline & $\begin{array}{l}\text { Left thoracotomy and neck } \\
\text { incision }\end{array}$ & 3 & 1.3 \\
\hline & $\begin{array}{l}\text { Right thoracotomy and neck } \\
\text { and abdominal incisions }\end{array}$ & 9 & 4 \\
\hline \multirow[t]{4}{*}{ Preoperative treatment } & Radiation & 4 & 1.9 \\
\hline & Chemotherapy & 21 & 10 \\
\hline & Radiation and chemotherapy & 7 & 3 \\
\hline & None & 184 & 85 \\
\hline \multirow{4}{*}{ Adjuvant therapy } & Radiation & 13 & 6 \\
\hline & Chemotherapy & 10 & 5 \\
\hline & Radiation and chemotherapy & 5 & 2 \\
\hline & None & 188 & 87 \\
\hline
\end{tabular}

status designates four groups of patients with distinctly different prognoses (Fig. 2, B).

The differences in overall survival are reflected in the median survivals shown in Table IV and in the disease-free survival curves shown in Fig. $3(A)$. The apparently large differences in disease-free survival between T1 through T2 N1 M0 and T3 N0 through N1 M0 tumors, and those between T1 through T2 N2 M0 and T3 N2 M0 tumors, must be viewed cautiously because the small number of patients in some of these TN subsets does not permit statistically valid comparisons (Fig. 3, B).

Overall survival was also analyzed according to the number of positive lymph nodes, irrespective of node location. Data concerning the total number of lymph nodes sampled were not available for 21 patients operated on during the first 2 years of this
Table III. Initial site of relapse according to tumor histologic characteristics

\begin{tabular}{lccc}
\hline & \multicolumn{2}{c}{ Tumor histology } \\
\cline { 2 - 3 } \multicolumn{1}{c}{ Site of relapse } & Adenocarcinoma & $\begin{array}{c}\text { Squamous } \\
\text { cell }\end{array}$ & $\begin{array}{c}\text { Total } \\
\text { patients }\end{array}$ \\
\hline Local & $14(19 \%)$ & $7(16 \%)$ & 22 \\
Regional & $14(19 \%)$ & $12(21 \%)$ & 26 \\
Distant & $35(48 \%)$ & $17(38 \%)$ & 53 \\
Local and regional & 5 & 5 & 10 \\
Local and distant & 2 & 2 & 4 \\
Regional and distant & 3 & 1 & 4 \\
Total & 73 & 44 & 119 \\
\hline
\end{tabular}

series, when such information was not always carefully noted in pathology reports. For the remaining 195 patients, the median number of lymph nodes sampled was 17 (range 1 to 57 nodes). Correspond- 


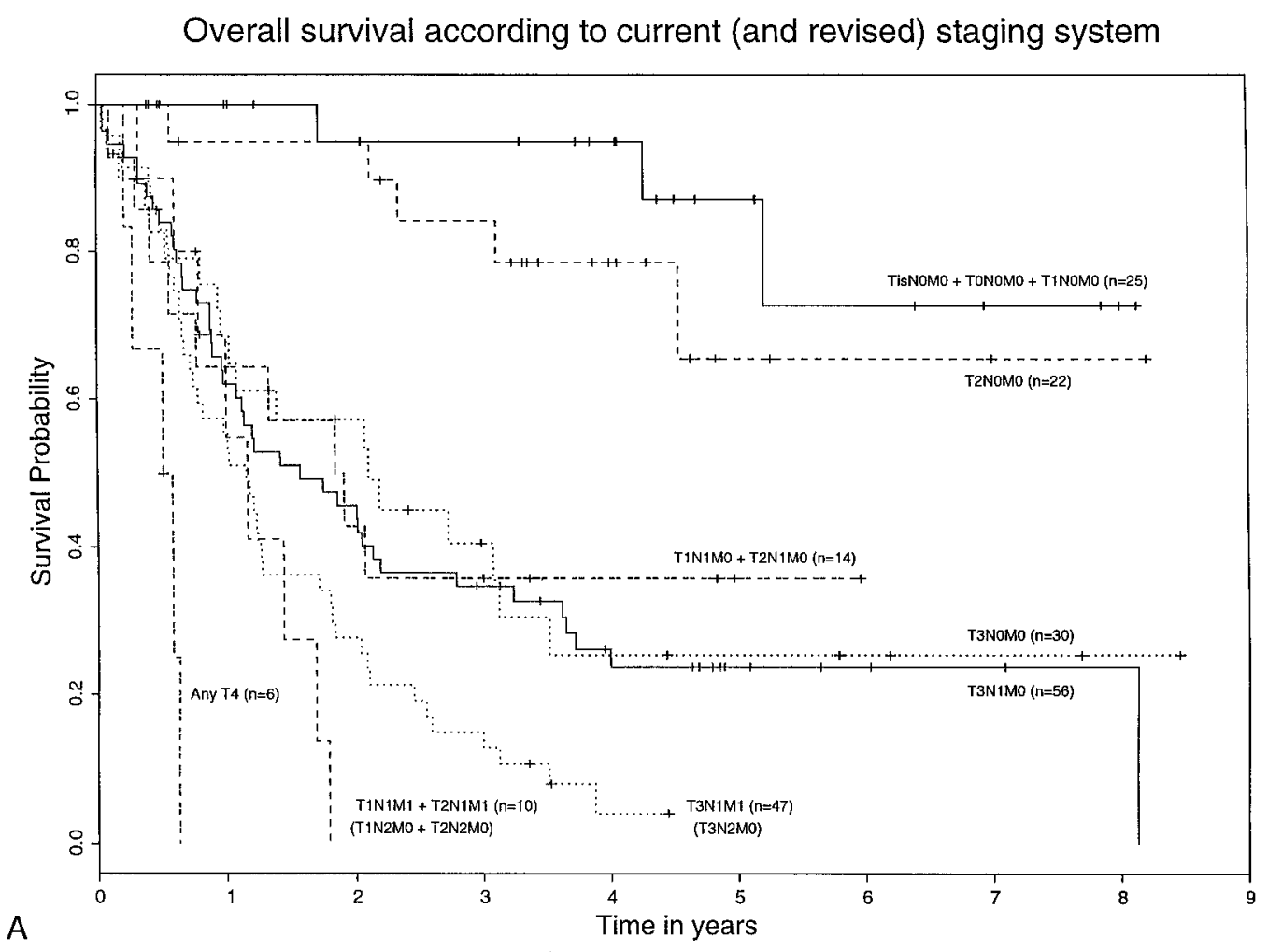

A

Overall survival according to current (and revised) staging system

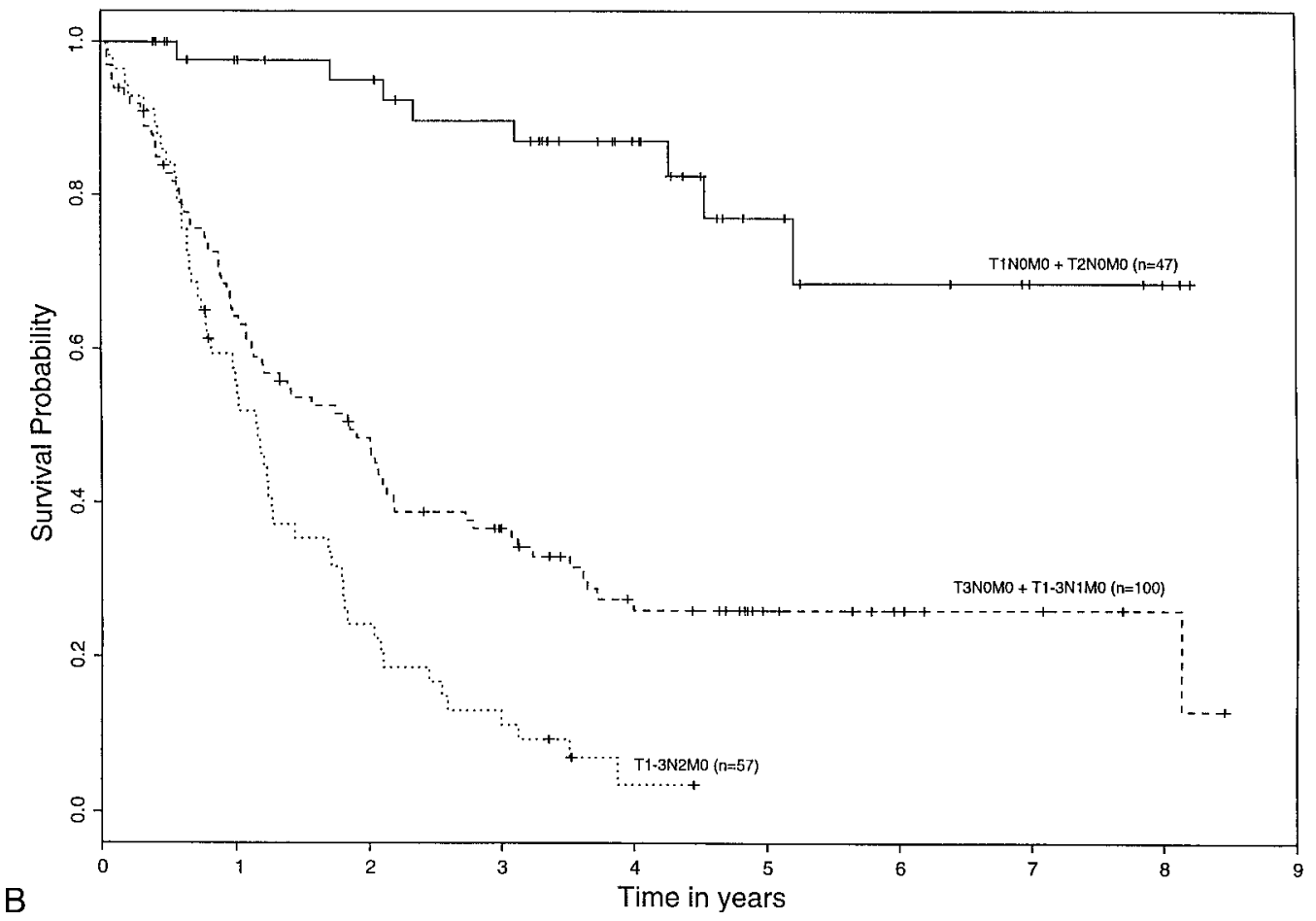

Fig. 2. A, Overall survival according to TNM stage. The revised staging system is noted in parentheses. B, Overall survival according to the newly proposed stage groupings with the revised TNM system. Table V describes stage groupings. Stage IV (T4, any N, M0 and any T, any N, M1) is not depicted. 
ing to the overall survival curves for T1 through T2 N0 M0 tumors, patients who had no positive nodes had a significantly better survival rate than did any other group (Fig. 4, $A$ ). There was no significant difference in overall survival among the patients who had one, two, or three positive nodes $(p=0.54)$, but there was a significant difference in survival between this overall group and patients who had four or more positive nodes $(p<0.001)$. Three groups of patients with distinctly different prognoses could thus be distinguished from this analysis: patients without positive nodes, patients with one to three positive nodes, and patients with four or more positive nodes (Fig. 4, B). The number of positive lymph nodes did not correlate with $\mathrm{N} 1$ versus N2 nodal status, indicating that this is a separate prognostic indicator. In a multivariate analysis, both TNM status and the number of positive nodes had a statistically significant impact on overall survival in patients with four or more positive nodes (hazard ratio 1.77); however, a score of one to three positive nodes was not an independent predictor of a worse survival (hazard ratio 0.93).

\section{Discussion}

The purpose of a cancer staging system is to predict survival on the basis of the anatomic extent of tumor. This information can then be used to direct therapy. For a staging system to be accurate and clinically useful, it must be based on data derived from the histologic type of tumor being staged. However, the current AJCC staging system for esophageal carcinoma is based primarily on retrospective data from the Japanese Committee for Registration of Oesophageal Carcinoma Cases, which focused on patients with squamous cell carcinoma of the cervical and thoracic esophagus. ${ }^{5,6}$ The adequacy of this system for esophageal carcinoma in western Europe and North America is questionable because most patients in western countries now have adenocarcinoma of the distal esophagus and gastroesophageal junction. However, it seems appropriate that adenocarcinomas of the gastroesophageal junction be staged along with tumors of the esophageal body, because data from our institution and others indicate that overall survival is not linked to tumor histologic characteristics or location. ${ }^{7-9}$

The staging system must also reflect parameters that have a strong prognostic influence for the tumor being staged. Although the extent of tumor invasion into the esophageal wall ( $\mathrm{T}$ descriptor) as currently described in the AJCC staging system
Table IV. Median survival and hazard ratio (risk of death) by TNM category

\begin{tabular}{lcc}
\hline \multicolumn{1}{c}{ TNM stage } & $\begin{array}{c}\text { Median survival } \\
(\text { mo })\end{array}$ & Hazard ratio \\
\hline T0 N0, Tis N0, T1 N0 & Not yet reached & 0.10 \\
T2 N0 M0 & Not yet reached & 0.20 \\
T3 N0 M0 & 25 & 0.87 \\
T1 N1 M0, T2 N1 M0 & 22.5 & 0.86 \\
T3 N1 M0 & 18.8 & 1.00 \\
T1 N2 M0, T2 N2 M0 & 13.9 & 2.09 \\
T3 N2 M0 & 13.8 & 1.76 \\
Any T4 & 6.5 & 6.63 \\
\hline
\end{tabular}

appears to be an important prognostic factor, the $\mathrm{N}$ descriptors in this system are less specific and less well validated. As a result, there is still controversy regarding regional (N1) versus distant (M1) nodal metastases. There is also no provision for the number of metastatic lymph nodes in the AJCC system, a criterion reported to be important in predicting survival. ${ }^{10-12}$

The designation of nonregional metastatic lymph nodes as M1 disease again stems from the Japanese data. The 5-year survival for 658 patients with positive lymph nodes outside of the regional nodal groups (M1) was only $5.2 \%$, compared with $16.8 \%$ for 1211 patients in whom only the regional nodes were positive. ${ }^{5,6}$ Little data exist in the western literature concerning survival with respect to the location of involved lymph nodes for patients with resected esophageal carcinoma. Clark and associates $^{13}$ reported on 43 patients with adenocarcinoma of the distal esophagus and gastroesophageal junction. Lymph nodes were stratified into four groups according to distance from the primary tumor. All patients underwent en bloc esophagectomy with radical lymphadenectomy. The location of involved lymph nodes did not influence survival, but the number of cases analyzed was small.

In accordance with the Japanese data, our study suggests that in cases where the lymph nodes are positive, the further the diseased lymph nodes are from the primary tumor, the worse the prognosis. However, even patients who had positive "N2" nodes according to our revised staging system appear to have a better survival than do patients with visceral metastases, who historically have a median survival of 4 to 6 months. ${ }^{14}$ Our findings are in agreement with those of Steup and colleagues, ${ }^{15}$ who studied 95 patients with carcinomas of the gastroesophageal junction and determined that patients with lymph node metastasis outside the re- 


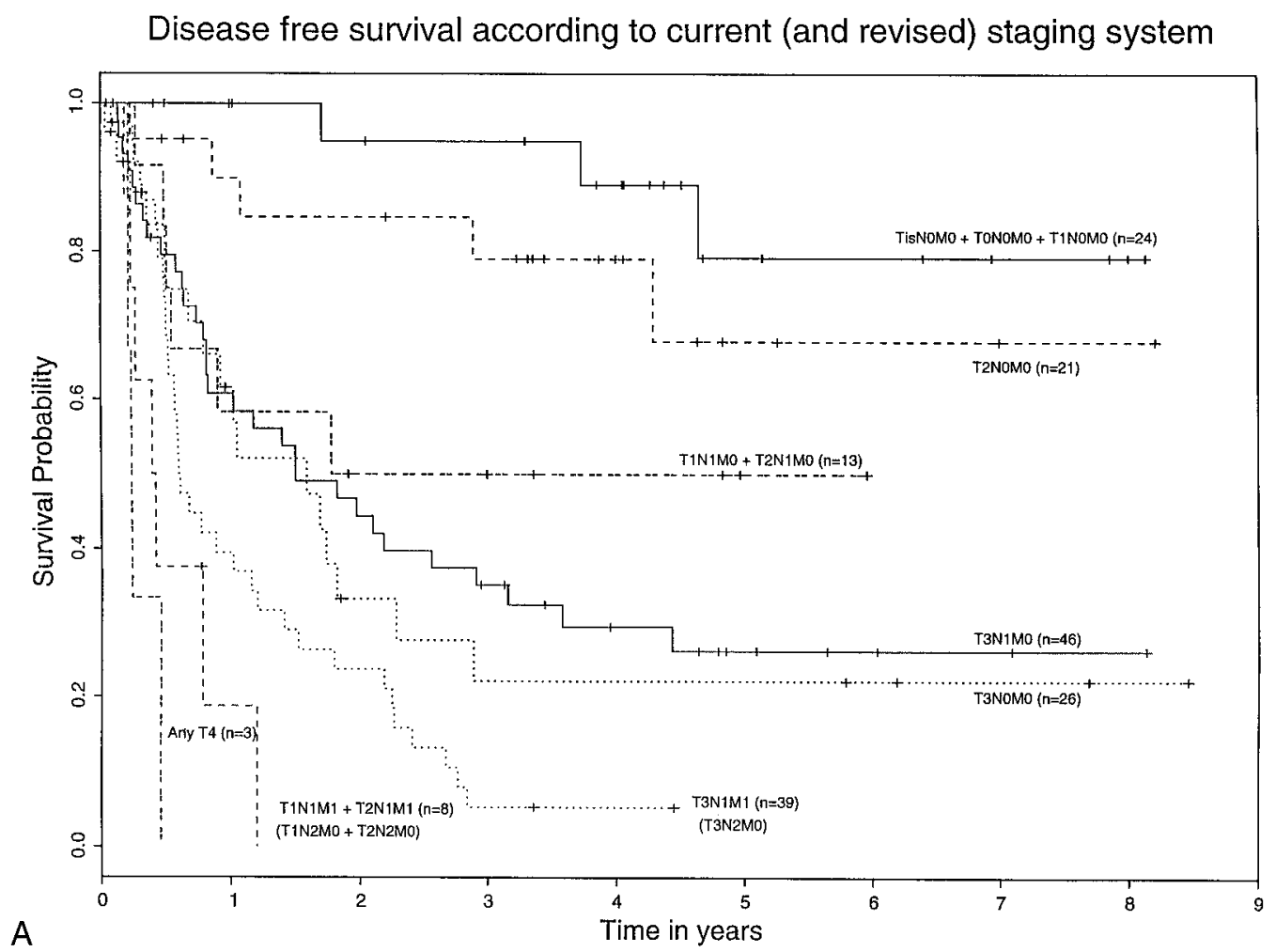

A

Disease free survival according to current (and revised) staging system

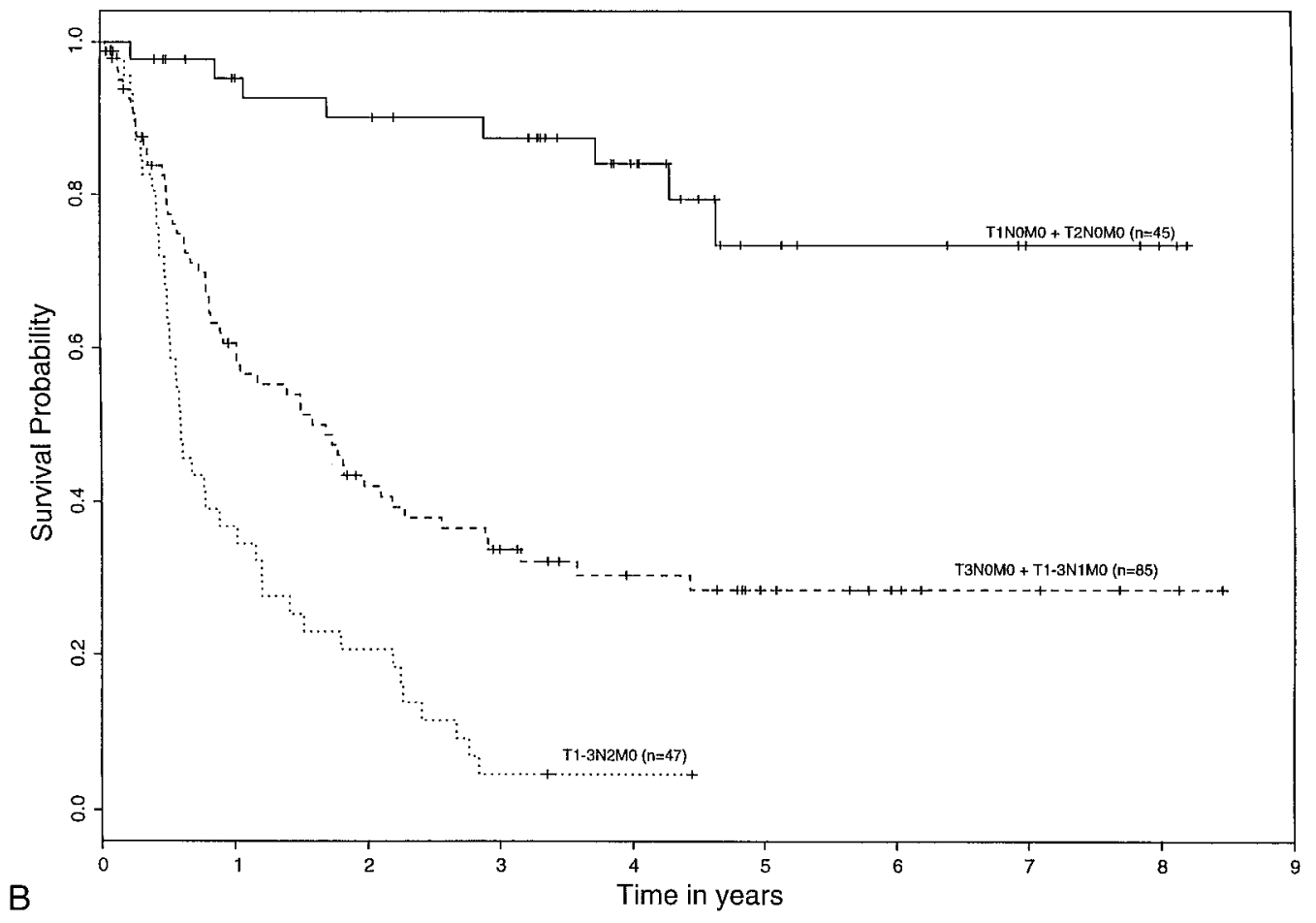

Fig. 3. A, Disease-free survival according to TNM stage. The revised staging system is noted in parentheses. B, Disease-free survival according to the newly proposed stage groupings with the revised TNM system. Table V describes stage groupings. Stage IV (T4, any N, M0 and any T, any N, M1) is not depicted. 


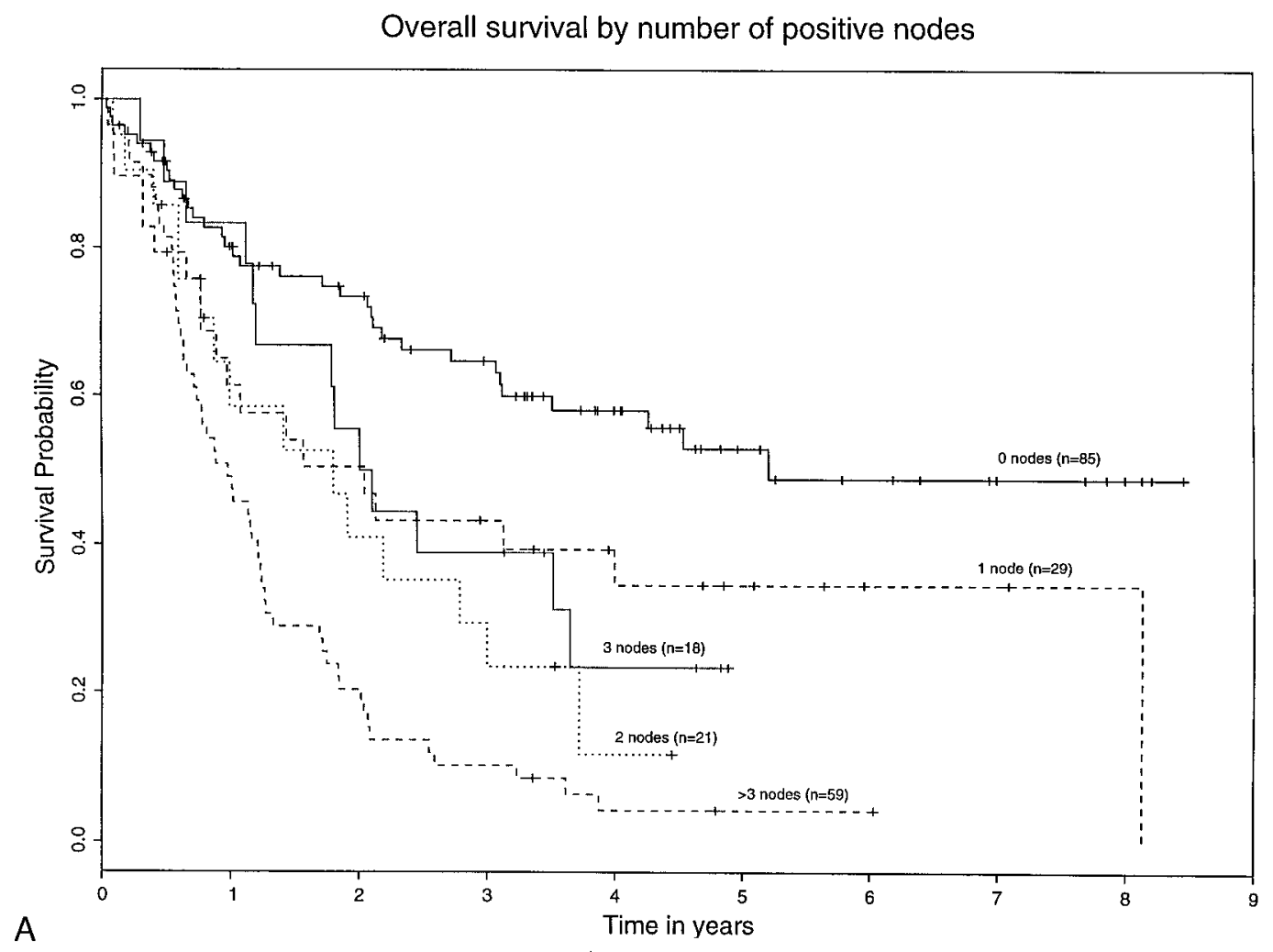

Overall survival by number of positive nodes

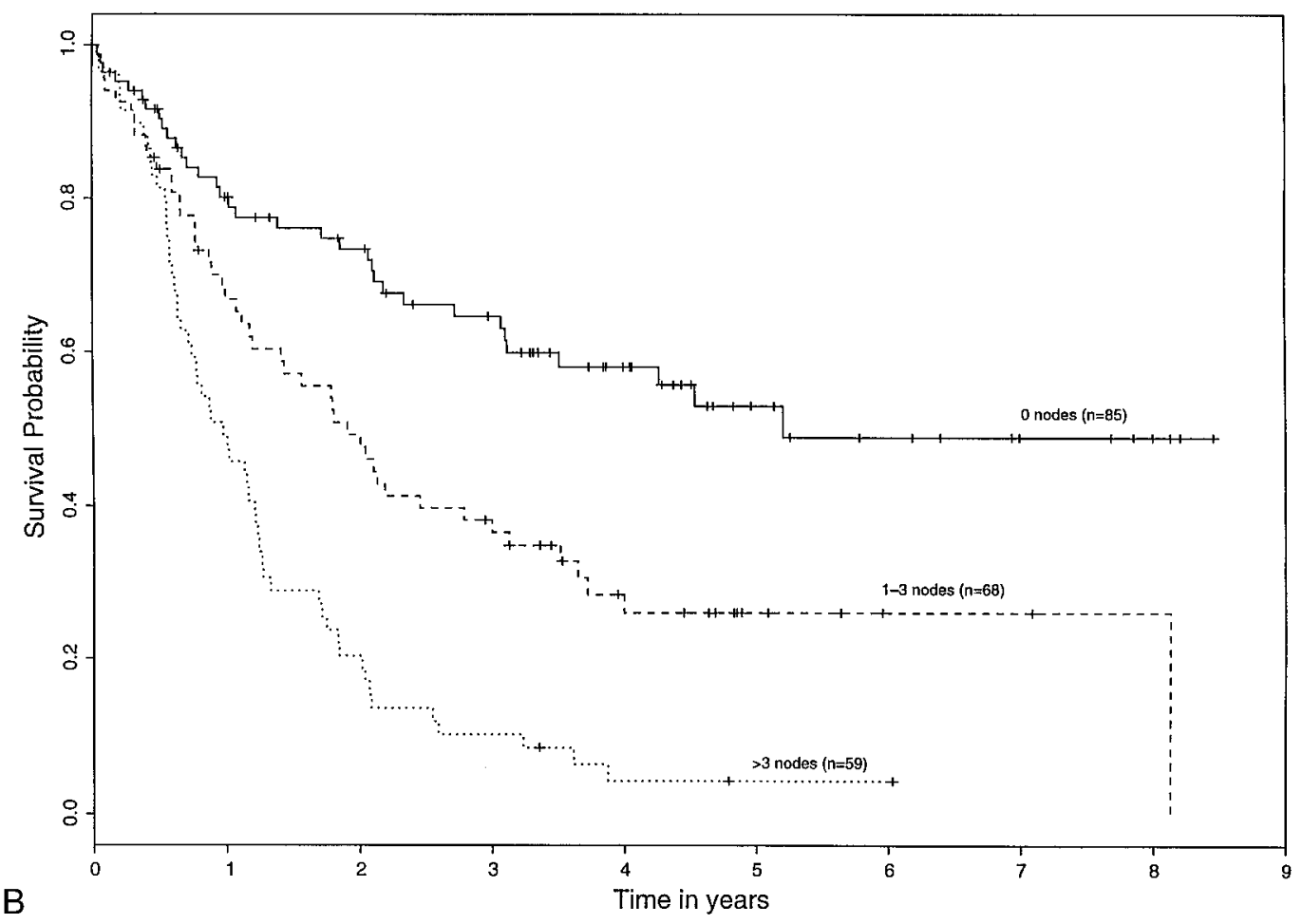

Fig. 4. A, Overall survival according to number of positive lymph nodes. B, Overall survival curves of patients with significantly different prognoses according to the number of positive lymph nodes. 
Table V. Current and proposed stage groupings for squamous cell carcinoma and adenocarcinoma of the esophagus

\begin{tabular}{cll}
\hline & $\begin{array}{c}\text { Current AJCC stage } \\
\text { grouping }\end{array}$ & \\
& \multicolumn{3}{c}{ Proposed grouping } \\
\hline Stage 0 & T0 N0, Tis N0 M0 & T0 N0, Tis N0 M0 \\
Stage I & T1 N0 M0 & T1 N0 M0 \\
& & T2 N0 M0 \\
Stage II* & & T3 N0 M0 \\
IIa & T2 N0 M0 & T1 N1 M0 \\
& T3 N0 M0 & T2 N1 M0 \\
IIb & T1 N1 M0 & T3 N1 M0 \\
Stage III & T2 N1 M0 & T1 N2 M0 \\
& T4 any N M0 & T2 N2 M0 \\
Stage IV & Any T, any N, M1 $\dagger$ & T3 N2 M0 \\
& & T4 any N M0 \\
& & Any T, any N, M1
\end{tabular}

*Subdivisions IIa and IIb are only applicable to the AJCC system. $\dagger$ Represents both visceral and distant nodal metastases.

$\ddagger$ Represents visceral metastases only.

gional nodes had a better prognosis after resection than did those with visceral M1 disease. As a result, these authors also advocate the creation of an N2 category according to nodal location with respect to the primary tumor site.

Both the number of positive lymph nodes and the ratio of positive lymph nodes to total number of nodes sampled are reported to influence survival after resection of esophageal carcinomas. ${ }^{10-12}$ Skinner and DeMeester ${ }^{10,12}$ incorporate an N2 category into their WNM staging system; this represents patients with more than four positive lymph nodes. A previous report from this institution also suggests that the number of diseased lymph nodes may influence survival. ${ }^{9}$ In the current study, the number of positive lymph nodes significantly influenced prognosis when four or more nodes were involved by metastatic disease. This finding needs further validation in prospective studies, but it may be useful as a stratification factor in future clinical trials.

Other authors have suggested that the staging system for esophageal carcinoma should be revised, including Killinger and associates ${ }^{16}$ who studied 345 patients undergoing resection of primary carcinoma of the esophagus. Our data are similar to theirs in the following respects. First, both studies report similar 5-year survivals for patients with T1 N0 M0 and T2 N0 M0 disease, suggesting that these two categories should be combined to form stage I. Second, the survival of patients with T3 N0 M0 disease in both studies more closely parallels that of patients with regional lymph node metastasis (N1).

Similarly, Ellis and colleagues ${ }^{17}$ reported on 265 patients who underwent resection for carcinoma of the esophagus. Again, patients with T1 N0 M0 tumors had a similar survival to those with T2 N0 M0 lesions. Additionally, their data concur with those of Skinner and coworkers ${ }^{8}$ and our own with respect to the absolute number of positive lymph nodes as an important prognostic indicator, suggesting that this criterion should be used to stratify patients in the N1 and N2 categories.

Although this study and those of the other authors suggest that revision of the current AJCC staging system for esophageal carcinoma is needed, these data must be interpreted with caution for the following reasons. First, in the ideal situation a radical lymphadenectomy should be performed to make nodal staging as accurate as possible. In this study, although most patients underwent transthoracic esophagectomy with extensive nodal sampling, systematic radical lymphadenectomies were not performed and some positive lymph nodes may not have been found. In the $21 \%$ of patients who had transhiatal esophagectomies, lymph node sampling was less extensive, with a greater potential for missing involved nodes. Second, the nature of the esophagectomy specimen can make pathologic evaluation and exact nodal location difficult. In some cases the esophagus is resected with most nodal tissue en bloc, leaving the classification of the lymph nodes to the discretion of the pathologist, who has never seen the specimen in situ. Also, the esophagus shortens appreciably after resection, which may confound nodal localization further.

Our experience suggests that it is appropriate to formulate a new staging classification applicable to both squamous cell and adenocarcinomas of the esophagus and gastroesophageal junction that more accurately reflects clinical outcome. Stage I would include both T1 N0 M0 and T2 N0 M0 tumors. Stage II would include disease limited to regional lymph nodes (T1 through T3 N1 M0) and also T3 N0 M0 tumors. Stage III consists of all T1 through T3 N2 M0 disease, reflecting the finding that patients with $\mathrm{N} 2$ disease, as defined here, have a worse prognosis than that of those with N1 disease but better than the historical outcome of patients with visceral metastases (M1). Stage IV comprises all T4 lesions and also visceral metastases (M1). Table V compares the proposed new system with the current AJCC staging system. Stratification for the number 
of positive lymph nodes, especially if four or more nodes are involved, appears to be important in detecting patients with a significantly worse prognosis. Such a revision of the AJCC staging classification will facilitate decisions about clinical care and will enhance the ability to select patients for entry into clinical trials for this difficult to treat malignancy.

We thank Marie Gregorio, RN, and Jackie Hanson, $\mathrm{RN}$, for their invaluable assistance in collecting data on patient follow-up, and Melody Owens for her outstanding assistance in data management and manuscript preparation.

\section{REFERENCES}

1. American Joint Committee on Cancer. In: Beahrs OH, Henson DE, Hutter RVP, Myers MH, editors. Manual for staging of cancer. 3rd ed. Philadelphia: JB Lippincott; 1988. p. 63-7.

2. Casson AG, Rusch VW, Ginsberg RJ, Zankowicz N, Finley RJ. Lymph node mapping of esophageal cancer. Ann Thorac Surg 1994;58:1569-70.

3. Kaplan EL, Meier P. Non-parametric estimation from incomplete observations. J Am Statist Assoc 1958;53:457-81.

4. Peto R, Peto J. Asymptotically efficient rank invariant test procedures. J R Statist Soc 1972;135:185-206.

5. The Japanese Committee for Registration of Esophageal Carcinoma. A proposal for a new TNM classification of esophageal carcinoma. Jpn J Clin Oncol 1985;14:625-36.

6. The Japanese Committee for Registration of Esophageal Carcinoma. Parameters linked to ten-years survival in Japan in resected cases of esophageal carcinoma. Chest 1989;96: 1005-11.

7. Heidl G, Langhans P, Mellin W, Bunte H, Grundmann E. Adenocarcinomas of the esophagus and cardia in comparison with gastric carcinoma. J Cancer Res Clin Oncol 1993;120: 95-9.

8. Clark GWB, Smyrk TC, Burdiles P, Hoeft SF, Peters JH, Kiyabu M. Is Barrett's metaplasia the source of adenocarcinomas of the cardia? Arch Surg 1994;129:609-14.

9. Lieberman MD, Shriver CD, Bleckner S, Burt M. Carcinoma of the esophagus: prognostic significance of histologic type. J Thorac Cardiovasc Surg 1995;109:130-9.

10. Skinner DB, Ferguson MK, Soriano A, Little AG, Staszak VM. Selection of operation for esophageal cancer based on staging. Ann Surg 1986;204:391-401.

11. Roder JD, Busch R, Stein HJ, Fink U, Siewert JR. Ratio of invaded to removed lymph nodes as a predictor of survival in squamous cell carcinoma of the oesophagus. Br J Surg 1994;81:410-3.

12. Hagen JA, Peters JH, DeMeester TR. Superiority of extended en bloc esophagogastrectomy for carcinoma of the lower esophagus and cardia. J Thorac Cardiovasc Surg 1993;106:850-9.

13. Clark GWB, Peters JH, Ireland AP, Ehsan A, Hagen JA, Kiyabu MT, et al. Nodal metastasis and sites of recurrence after en bloc esophagectomy for adenocarcinoma. Ann Thorac Surg 1994;58:646-54.
14. Anderson LL, Lad TE. Autopsy findings in squamous cell carcinoma of the esophagus. Cancer 1982;50:1587-90.

15. Steup WH, De Leyn P, Deneffe G, Van Raemdonck D, Coosemans W, Lerut T. Tumors of the esopahgogastric junction: long term survival in relation to the pattern of lymph node metastasis and a critical analysis of the accuracy or inaccuracy of pTNM classification. $\mathrm{J}$ Thorac Cardiovasc Surg 1996;111:85-95.

16. Killinger WA, Rice TW, Adelstein DJ, Medendorp SV, Zuccaro G, Kirby TJ. Stage II esophageal carcinoma: the significance of T and N. J Thorac Cardiovasc Surg 1996;111: 935-40.

17. Ellis FH, Watkins E, Krasna MJ, Heatley GJ, Balogh K. Staging of carcinoma of the esophagus and cardia: a comparison of different staging criteria. J Surg Oncol 1993;52:231-5.

\section{Discussion}

Dr. Tom R. DeMeester (Los Angeles, Calif.). The business of staging esophageal carcinoma is certainly now undergoing review. A number of articles have indicated that at least the number of nodes involved is important and has not previously been fully appreciated. This article takes on particular emphasis in the era where the number of adenocarcinoma associated with Barrett esophagus is rising exponentially, as indicated in your presentation.

My questions focus on the alteration of the staging methodology that you propose, namely whether survival is affected by $\mathrm{T} 1$ and $\mathrm{T} 2$ tumors, the number of nodes involved, and the location of the involved nodes. First, your article and others preceding it all agree that the number of nodes is important, that there is a difference between having none, five or fewer, or more than five nodes involved. What about the ratio of positive nodes to negative nodes? There has been a discussion in the literature that the ratio of involved to uninvolved removed is important in prognosis; could you comment on this, or do you have any data that would support or refute this contention?

Dr. Korst. I do not have data pertaining to the number of negative nodes.

Dr. DeMeester. My second question has to do with the distance that a positive node is from the primary tumor and its effect on survival. Is this really an important phenomenon, or is it just that a patient who has distant nodes involved is more likely to have a greater number of nodes involved? In that regard, I wonder, did you have situations where there were fewer than five nodes involved but that they were some distance removed from the primary tumor, that is, in the sites that you identified, with a negative effect on prognosis?

Dr. Korst. There were situations where positive nodes were found in distant nodal stations in the absence of positive nodes immediately adjacent to the primary tumor. In addition, there were situations where many positive nodes were found adjacent to the primary tumor, but no distant nodes were positive. There thus did not seem to be a correlation between the number of positive nodes and the likelihood of distant nodal disease. Distance from the primary tumor was an independent prognosticator in the patients.

Dr. DeMeester. Did you say that a few positive nodes but distantly removed correlated with survival? 
Dr. Korst. No.

Dr. DeMeester. So you do not think that the distance an involved node is from the primary tumor is an independent factor in prognosis?

Dr. Korst. No; I mean that I believe distance from the primary tumor is an independent factor.

Dr. DeMeester. Do you know the number of nodes that were removed per patient?

Dr. Korst. I do not have the figures concerning the total number of lymph nodes removed. However, I can elaborate a little bit on what is typically done during an esophagectomy. As I said, most procedures were transthoracic esophagectomies, and extensive mediastinal and abdominal lymph node sampling was performed; however, a radical, two-field or three-field lymphadenectomy is not routinely done.

Dr. DeMeester. In that case, do you think that the data you have are adequate for your analysis and recommendations?

Dr. Korst. I think that the extensive node sampling that was performed in those patients was adequate for the drawing of some preliminary conclusions. However, I agree that the more radical the lymphadenectomy, the more accurate the staging will be.

Dr. DeMeester. My third question focuses on the reports of histochemical studies done on lymph nodes, that is, nodes that are normal by histologic examination but are positive for metastatic tumor on histochemical analysis with monoclonal antibody to identify epithelial cell remnants. Some reports say that histochemically positive nodes have an effect on survival. Could you comment on whether these studies should be considered before we begin to revamp our staging methodology of this disease?

Dr. Korst. If it affects survival, then perhaps it should be considered as part of the staging process.

Dr. DeMeester. One area that I thought you may have had the data to address but did not in the article or the presentation is whether there is a difference in survival between T1 and T2 tumors. For the sake of the audience, I should define a T1 tumor. A T1 tumor is a tumor that extends beyond the muscularis mucosa into the submucosa.

Dr. Korst. Right.

Dr. DeMeester. And it differs from a T2 tumor in that a T2 tumor extends into the muscularis propria. There has been discussion that this difference is not of practical significance; that is, they have a similar effect on survival. Have you analyzed your specimens in an effort to identify tumors that were limited by the muscularis mucosa, those that extended into the submucosa, and those that extended into the muscularis propria?

Dr. Korst. Unfortunately, we didn't break down the tumors into mucosal versus submucosal, but obviously that is a very heated area and it would be worth doing.

Dr. DeMeester. I think such information would be of value, especially in the area of surveillance for early disease.
My last question varies a bit from the staging, but of course my interest was piqued by the fact that you had a high local recurrence rate after your resections. In the article, you reported a $45 \%$ to $50 \%$ local recurrence rate. Do you think a more extensive esophagectomy and gastrectomy with an en bloc resection would have reduced the local recurrence rate?

Dr. Korst. Of a total of 119 patients who had recurrent disease, 22 had local recurrences and 53 had distant metastatic disease. With this high rate of distant failure, it intuitively seems unlikely that a more radical resection would be of benefit.

Dr. James B. D. Mark (Stanford, Calif.). As you know, there is a new proposed staging for lung cancer that separates some node-positive from node-negative disease. Right now in lung cancer, a single stage includes T3 N0 and $\mathrm{T} 2 \mathrm{~N} 2$. You have again included in a single stage T3, N0 and T1 through T2 N1. Do you think that you might want to divide those into another stage, or a substage, so that in the future you can separate all N0 cancers from any with nodal involvement?

Dr. Korst. A close look at the slide with the survival curves shows that with the exception of the group of patients with T3 N0 tumors, the staging system broke down so that N0 was stage I, N1 was stage II, and N2 was stage III. If you look at the survival in the T3 N0 group, there really was no difference between that group and the rest of the patients with $\mathrm{N} 1$ disease. However, a significant difference in survival is present between the T3 N0 group and the rest of the N0 patients. It would be great if we could group T3 N0 into stage I, because it would make it even that much simpler, but the data do not allow us to do that.

Dr. Mark. I could not process that entire multicolored slide, but the one that followed it with the three lines I could handle. In the middle one in was the N1. You know what I am talking about?

Dr. Korst. Right. I know what you are talking about.

Dr. Mark. Second, you have accentuated the importance of numbers of nodes involved, yet nowhere in the staging system is that taken into account. I realize that this might be difficult, and Dr. DeMeester has addressed several questions about how many nodes you removed and what percentage of nodes were removed and so forth, but should that be an important part of the staging system?

Dr. Korst. I think it should be. The problem with our database is that some of those subgroups, specifically those of more superficial tumors that are node positive, do not have many patients in them. With the small number of patients, it is difficult to make statistically valid comparisons with respect to number of nodes.

Dr. Mark. I just encourage you to be a little more aggressive in operating on patients with cancer of the esophagus. You are leaving too many not operated on, I think. 